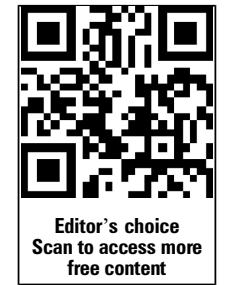

- Additional material is published online only. To view please visit the journal online (http://dx.doi.org/10.1136/ jclinpath-2014-202352).

For numbered affiliations see end of article.

\section{Correspondence to \\ Dr Umberto Gianelli, Hematopathology Service, Division of Pathology, Department of Pathophysiology and Transplantation, University of Milan, IRCCS Ca' Granda- Maggiore Policlinico Hospital Foundation, Via Francesco Sforza 35, Milano 20122, Italy; \\ umberto.gianelli@unimi.it}

CA and UG contributed equally.

Received 14 April 2014 Accepted 21 May 2014 Published Online First

9 June 2014
CrossMark

To cite: Augello C, Gianelli U, Savi F, et al. J Clin Pathol 2014;67 697-701.

\title{
MicroRNA as potential biomarker in HCV-associated diffuse large B-cell lymphoma
}

\author{
Claudia Augello, ${ }^{1}$ Umberto Gianelli, ${ }^{2}$ Federica Savi, ${ }^{3}$ Alessia Moro, ${ }^{3}$ \\ Emanuela Bonoldi, ${ }^{4}$ Marcello Gambacorta, ${ }^{5}$ Valentina Vaira, ${ }^{6}$ Luca Baldini, ${ }^{7}$ \\ Silvano Bosari ${ }^{8}$
}

\begin{abstract}
Aims To identify molecular characteristics to hepatitis C virus (HCV)-associated diffuse large B-cell lymphoma (DLBCL) through a comprehensive miRNAs expression profiling.

Methods In this study, miRNA profiles were obtained from 37 patients with DLBCLs and 60 patients with reactive lymph nodes, equally distributed according to HCV presence. Germinal centres, from reactive lymph nodes were used as controls. Clinical features at presentation were available for all patients.

Results A set of 52 miRNAs define a signature for HCV-associated DLBCL. Importantly, decreased expression of miR-138-5p and increased expression of miR-147a, miR-147b and miR-511-5p in HCV DLBCL was found to be a poor prognostic factor for HCVpositive DLBCL patients.
\end{abstract}

Conclusions These data reveal molecular differences in diffuse DLBCL patients according to HCV presence, potentially useful as novel prognostic or therapeutic biomarkers.

\section{BACKGROUND}

Hepatitis $\mathrm{C}$ virus (HCV) infection frequently leads to chronic hepatitis and is a major cause for liver cirrhosis, hepatocellular carcinoma (HCC) and extra-liver haematological manifestations, such as type II mixed cryoglobulinemia. ${ }^{1}$ Epidemiologic studies, therapeutic approaches and experimental data have already established an association between HCV infection and B-cell non-Hodgkin lymphoma (NHL) development. ${ }^{2}{ }^{3}$ Higher prevalence of HCV positivity was observed in marginal zone lymphomas, chronic lymphocytic leukaemia, lymphoplasmacytic lymphoma and diffuse large B-cell lymphoma (DLBCL). ${ }^{2}$

DLBCL is a highly heterogeneous group of B-cell lymphomas. Gene-expression profiling identified three major subgroups of DLBCL: germinal centre B-cell-like DLBCL, activated B-cell-like DLBCL, and primary mediastinal large B cell lymphoma. ${ }^{4}$ DLBCL subgroups are characterised by different clinical features, therapy response and prognosis. ${ }^{5}$ None of the morphologic features can distinguish HCV-associated NHL from negative ones. ${ }^{6}$ Compared with the latter, HCV-positive DLBCL patients often present more elevated lactate dehydrogenase levels, high hepatic toxicity of chemotherapy and unfavourable outcome. ${ }^{7}$

MicroRNAs (miRNAs) are small single-stranded, non-coding RNAs, that negatively regulate gene expression by targeting specific mRNAs. A bulk of data has already established an important role for miRNAs among factors involved in cancer pathogenesis, ${ }^{8}$ viral infection ${ }^{9}$ and virus carcinogenesis. ${ }^{10}$

In DLBCL studies, miRNAs are emerging as clinically important molecular markers: the role of miR155 as oncomiR in pathogenesis and aggressiveness of DLBCL is clearly defined. ${ }^{11}$ Recent studies identified a set of miRNAs expression (miR-222, miR-181a, miR129-5p and miR-18a) associated with prognosis in DLBCL patients. ${ }^{12}$ However, no data are known to be available on miRNAs expression in $\mathrm{HCV}$-associated DLBCL so far.

In this study, we aimed to analyse molecular characteristics peculiar to $\mathrm{HCV}$-associated DLBCL through a comprehensive miRNAs expression profiling.

\section{PATIENTS AND METHODS \\ Patients}

Ninety-seven formalin-fixed and paraffin-embedded tissues, collected between 2001 and 2009, from three institutions (San Paolo Hospital, IRCCS Ca' Granda-Maggiore Policlinico Hospital Foundation and Niguarda Ca' Granda Hospital, Milan, Italy) were available for the study. Tissue samples included: (1) DLBCL from patients with HCV chronic infection ( $\mathrm{n}=19),(2)$ DLBCL from patients without $\mathrm{HCV}$ infection $(\mathrm{n}=18)$, (3) reactive lymph nodes from HCV serologically positive patients $(n=30),(4)$ reactive lymph nodes from HCV serologically negative patients $(n=30)$. All the cases were reviewed by two expert pathologists (UG and FS), and DLBCL were classified according to the updated WHO classification. ${ }^{13}$ Germinal centres, from reactive lymph nodes were laser-assisted microdissected (LMD6000, Leica Microsystems, Milan, Italy) (figure 1), as previously described, ${ }^{14}$ and used as controls.

Clinical and pathological features of DLBCL patients are detailed in table 1 . The follow-up period ranged from 3 to 90 months (average 27 months). At the last follow-up, 20 patients (51\%) were deceased for lymphoma disease. All the patients gave their informed consent.

\section{RNA purification and miRNA profiling}

RNA was isolated and purified using Master Pure RNA purification kit (Epicentre Biotechnologies, Madison Wisconsin, USA) according to the manufacturer's protocol. Total RNA from germinal centre B cells of each 10 reactive lymph nodes were combined; so six RNA pools were obtained (3 from $\mathrm{HCV}^{+}$and 3 from $\mathrm{HCV}^{-}$patients) and used as 

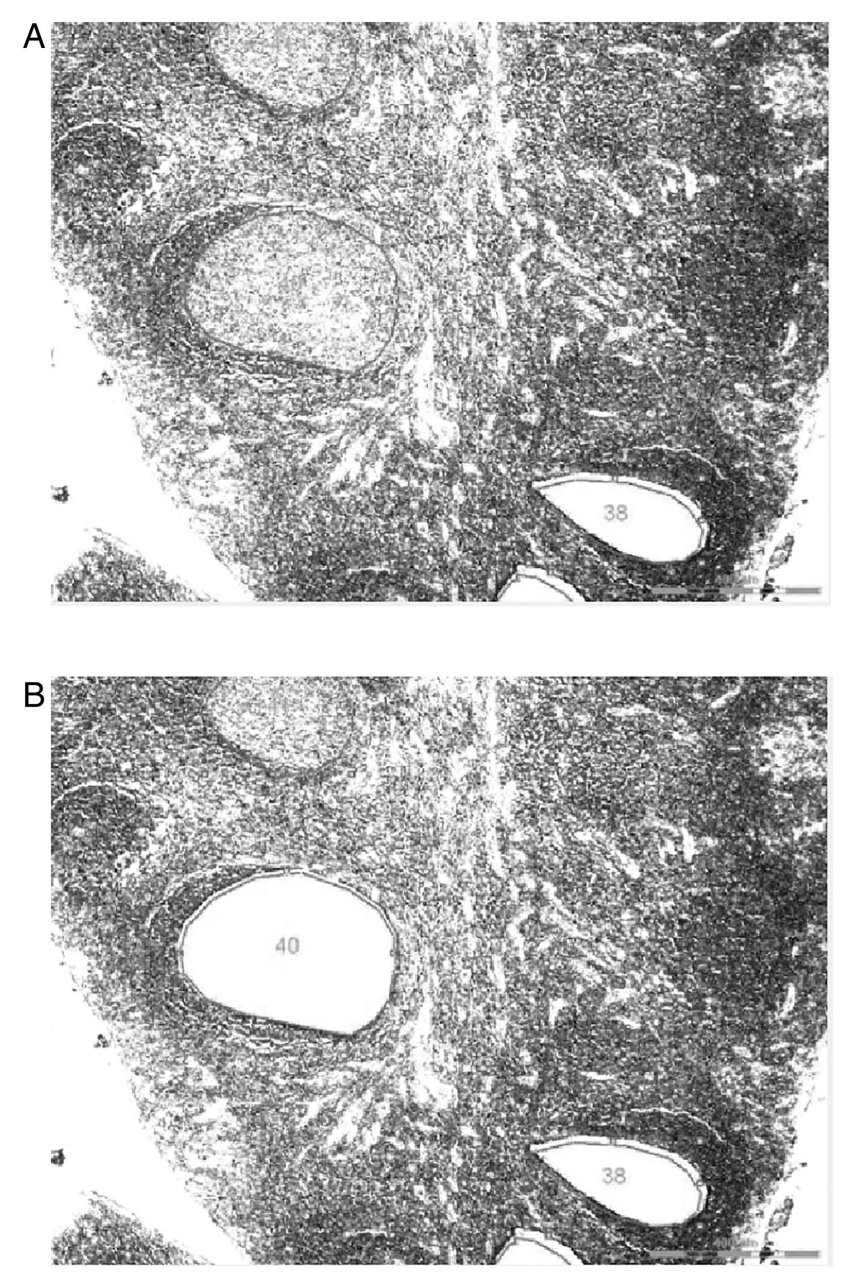

Figure 1 Isolating germinal centre by laser-assisted microdissection. Germinal centres were morphologically identified on haematoxylin-stained sections and purified by laser-assisted microdissection (LMD).

control. Differently, each tissue sample of DLBCL patients has been analysed individually.

For each DLBCL tissue sample and germinal centre B cells pools, $300 \mathrm{ng}$ of total RNA was used to reverse transcription (RT) and preamplification reactions (Megaplex Primer Pools, Human set V.2.0: Applied Biosystems by Life Technologies, Carlsbad, California, USA). The RT and preamplification reactions were performed in multiplex modality according to the manufacturer's protocol. TaqMan MicroRNA arrays (cards A and B, V.2.0) were used to profile the expression of 664 miRNAs in lymphoid tissues.

Instrument raw data were $C_{t}$ (threshold cycle) converted by Sequence Detection System (SDS) 1.2 software (Applied Biosystem, Foster City, California, USA). All reagents, instrument and software were provided by Applied Biosystems (Life Technologies Foster City, California, USA).

Only miRNAs with $\mathrm{C}_{\mathrm{t}}$ values $<35$ in more than two samples were transformed in relative quantities (RQ) using the geometrical mean of the reference snoRNAs as normalisation factor and the $2^{-\Delta \mathrm{Ct}}$ formula. RNU48, RNU44 were used as reference snoRNAs in the arrays.

\section{Statistical analysis}

For statistical analysis of the expression profiles data, the miRNA RQs were median normalised and log2-transformed.
Table 1 Pathological and clinical characteristics of DLBCL patients

\begin{tabular}{|c|c|c|}
\hline Pathological feature & DLBCL $\mathrm{HCV}^{+}$ & DLBCL HCV ${ }^{-}$ \\
\hline Total & 19 & 18 \\
\hline Mean age in year, value (range) & $67(41-83)$ & 70 (32-91) \\
\hline Male sex, number of cases $(\%)$ & $12(63)$ & $11(61)$ \\
\hline HCV-RNA virus, number of cases (\%) & $19(100)$ & NA \\
\hline HCV-Ab positive ${ }^{*}$, number of cases (\%) & $19(100)$ & NA \\
\hline HCV- genotype 1, number of cases (\%) & $5(26)$ & NA \\
\hline HCV- genotype 2, number of cases (\%) & $14(74)$ & NA \\
\hline Bone marrow infiltration, number of cases (\%) & $6(31)$ & $4(22)$ \\
\hline Liver involvement number of cases (\%) & $4(21)$ & $1(5)$ \\
\hline Splenomegaly, number of cases (\%) & $5(26)$ & $2(11)$ \\
\hline \multirow[t]{2}{*}{ Germinal centre subtype number of cases (\%) } & GCB: 5 (26) & GCB: 5 (28) \\
\hline & No-GCB: 14 (74) & No-GCB: 13 (72) \\
\hline \multirow[t]{2}{*}{ Stage, number of cases (\%) } & I-II: 10 (52) & I-II: $11(61)$ \\
\hline & III-IV: 9 (48) & III-IV: 7 (39) \\
\hline \multirow{2}{*}{$\begin{array}{l}\text { International Prognostic Index, } \\
\text { number of cases (\%) }\end{array}$} & $1-2: 11(58)$ & 1-2: $11(61)$ \\
\hline & 3-4: $8(42)$ & 3-4: 7 (39) \\
\hline Follow up period: mean (range) & $28(3-90)$ & $23(3-70)$ \\
\hline Death, number of cases (\%) & $11(61)$ & $9(50)$ \\
\hline
\end{tabular}

For samples clustering log2-transformed data were imported in dChip software (DNA-Chip Analyser, http: ||www.dchip.org). Relative abundance of miRNAs was standardised by software preprocessing steps (subtracting its mean and dividing the result by its SD) and hierarchical clustering analysis was then performed.

Class comparison and class prediction functions from BRB-Array Tool software (V.3.8.1, http://inus/nci.nih.gov/ BRB-ArrayTools.html) were used in supervised analysis. Before supervised analyses were approached, miRNAs were filtered according to two criteria: a variation of the expression level of at least 2.5 -fold from the median value in at least $20 \%$ of the samples, and an associated $\mathrm{p}$ value $<0.01$. Two hundred and nine miRNAs passed the filter.

For class comparison, the t test was used as univariate statistics for each pairwise comparison. For class prediction, the featured selection criterion for a miRNA to be included in the classifier was a different expression between the classes of at least $\mathrm{p}<0.01$ significant level. To compute the misclassification rate, the leave-one-out cross-validation method was used.

The miR target lists were generated used TarBase V.6.0, a database of experimentally verified. ${ }^{15}$ To investigate the predicted functional implications of selected miRNAs on biological and cellular pathways, we used the Diana mirPath web server application V.2.0 (http://diana.cslab.ece.ntua.gr/pathways/), applying miR target lists generated by Tarbase 6.0 .

Correlations of miRNA expression in $\mathrm{HCV}$-associated DLBCL with clinicopathological features were analysed by ANOVA and a $p$ value lower than the threshold limit of $p<0.01$ were considered significant.

The overall survival curves for HCV-associated DLBCL patients were plotted using the Kaplan-Meier method. Patients were categorised in two groups according to the median of miRNA expression data. The log rank test was used to detect significant differences between patient curves, and $\mathrm{p}<0.05$ was considered statistically significant. 
Figure 2 Unsupervised analysis of microRNA in diffuse large $B$-cell lymphoma (DLBCL) and germinal centre $B$ cells. Hierarchical clustering of 542 miRNAs distinguish DLBCL samples from germinal centre $B$ cells (lymph node, $n=6 / 11,54 \%, p<0.001$ by Fisher's exact test). Clustering was performed with average linkage and 1-correlation as distance metric.

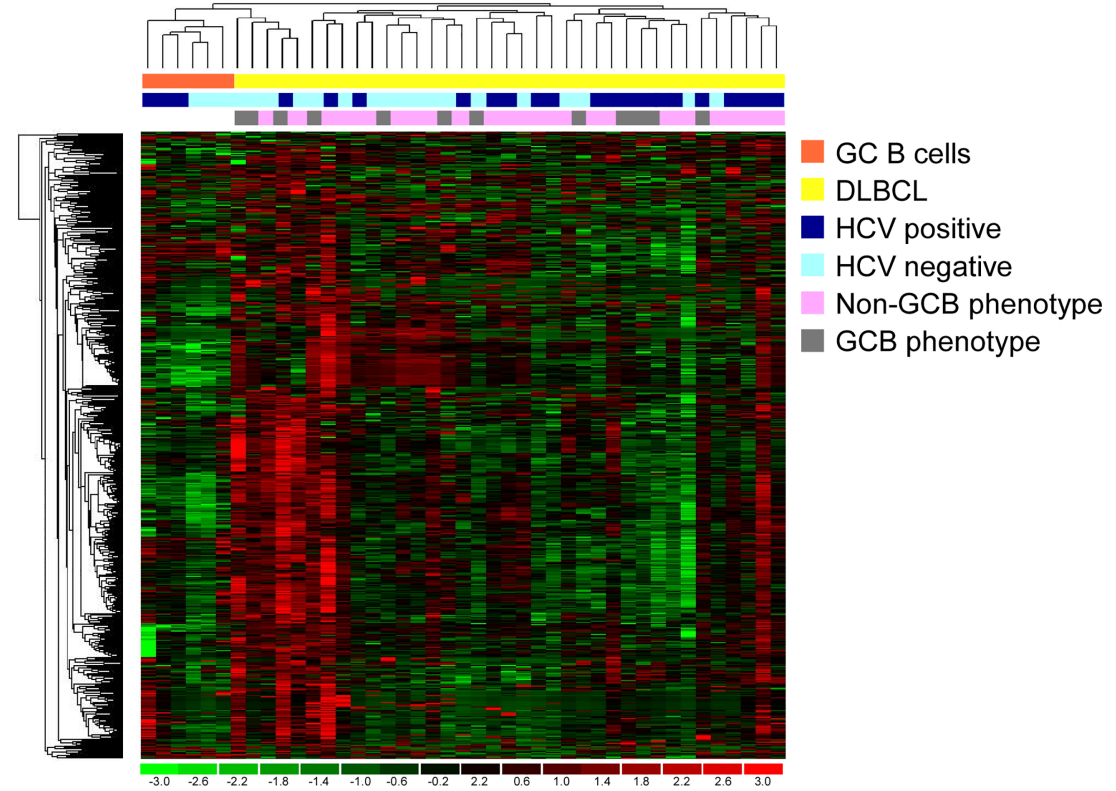

\section{RESULTS}

miRNA profiles distinguishes DLBCL from germinal-centre B cells

A panel of 542 out of 664 mature miRNAs (81\%) has been successfully detected in the analysed samples. To assess whether $\mathrm{HCV}$ infection in lymphoid tissues could be distinguished at a molecular level by miRNA expression profile, an unsupervised hierarchical cluster analysis was performed. The analysis was able to differentiate DLBCL from germinal-centre B cell, in $\mathrm{HCV}$-positive and $\mathrm{HCV}$-negative patients (figure 2). Considering only DLBCL, miRNA expression could not discriminate between infected patients and controls.

\section{Fifty-two miRNAs define a signature for HCV-associated DLBCL}

In $\mathrm{HCV}$-positive patient groups, we analysed differences between the DLBCL and germinal centre B cells. Two prediction tests (Class comparison and class prediction analyses) of BRB-ArrayTools were used to identify the classifier signature with the lowest misclassification error. The algorithms identified 52 miRNAs differentially expressed in DLBCL, compared to non-pathological B cells: 35 and 17 miRNAs were overregulated and under-regulated, respectively (see online supplementary table S1).

In order to identify the transcripts that might be targeted by differentially regulated miRNA in HCV-associated DLBCL, tarbase, a database containing experimentally verified $\mathrm{miR}$ interaction information, were used. Twenty-one out of 52 differentially regulated miRNAs showed validated target in tarbase. Analysis of Kyoto
Encyclopaedia of Genes and Genomes (KEGG) pathway database of these twenty-one validated targets were performed: the most affected pathway, modulated by at least one of the identified miRNAs, were PI3K-Akt signalling, cancer, HTLV-1 infection and viral carcinogenesis, with 23, 23, 18 and 17 genes, respectively (see online supplementary table S2).

\section{miRNA distinguished HCV-associated DLBCL compared to No-infected DLBCL}

To identify which miRNAs are significantly deregulated in $\mathrm{HCV}$-associated DLBCL tumorigenesis, the pairwise comparisons were made between HCV-positive and HCV-negative DLBCL. We detected 6 and 14 miRNAs over-regulated and under-regulated, respectively, in $\mathrm{HCV}$-associated DLBCL with respect to non-infected ones (see online supplementary table S3).

Moreover, we hypothesised that a miRNA likely could be highly involved in HCV-associated DLBCL tumorigenesis if it is deregulated in the two pairwise comparisons: $\mathrm{HCV}^{+}$DLBCL vs germinal centre $\mathrm{B}$ cells and $\mathrm{HCV}^{+}$DLBCL versus $\mathrm{HCV}^{-}$ DLBCL. The combinatorial strategy enabled the identification of three miRNAs significantly deregulated in $\mathrm{HCV}$-associated DLBCL: miR-20b-3p, miR-363-5p and mir-424-3p (table 2).

\section{miRNAs expression is correlated with clinical and} pathological features in HCV-associated DLBCL

A significant correlation was found between high miR-576-5p, miR-129-3p level, low miR-522, miR-512-3p level and germinal centre B cell-like DLBCL immunophenotype. Moreover, low

Table 2 MicroRNA differently expressed in HCV-associated DLBCL versus DLBCL and versus HCV-positive germinal centre B cells

\begin{tabular}{|c|c|c|c|c|c|c|c|}
\hline miR name & $\begin{array}{l}\text { Genomic } \\
\text { location* }\end{array}$ & $\begin{array}{l}\text { HCV-associated } \\
\text { DLBCL }\end{array}$ & DLBCL & $\begin{array}{l}\mathrm{HCV}^{+} \mathrm{B} \\
\text { cells }\end{array}$ & $\begin{array}{l}\mathrm{FC} \mathrm{HCV}^{+} \mathrm{DLBCL} / \\
\text { DLBCL }\end{array}$ & $\begin{array}{l}\text { FC HCV }{ }^{+} \mathrm{DLBCL} / \mathrm{HCV}^{+} \mathrm{B} \\
\text { Cells }\end{array}$ & $\begin{array}{l}\text { Number of validated } \\
\text { target } t\end{array}$ \\
\hline miR-363-5p & Xq26.2 & 0.33 & 3.27 & 24.99 & 0.10 & 0.01 & - \\
\hline miR-20b-3p & Xq26.2 & 0.22 & 1.57 & 3.41 & 0.14 & 0.06 & - \\
\hline miR-424-3p & Xq26.3 & 0.57 & 2.21 & 0.028 & 0.26 & 20.36 & - \\
\hline
\end{tabular}

The geometrical mean of the miRNA expression is provided.

${ }^{*}$ According to miRbase (http://www.mirbase.org/).

†According to tarbase 6.0 (http://diana.imis.athena-innovation.gr/).

$\mathrm{DLBCL}$, diffuse large B-cell lymphoma; $\mathrm{FC}$, fold-change; $\mathrm{HCV}$, hepatitis $\mathrm{C}$ virus. 

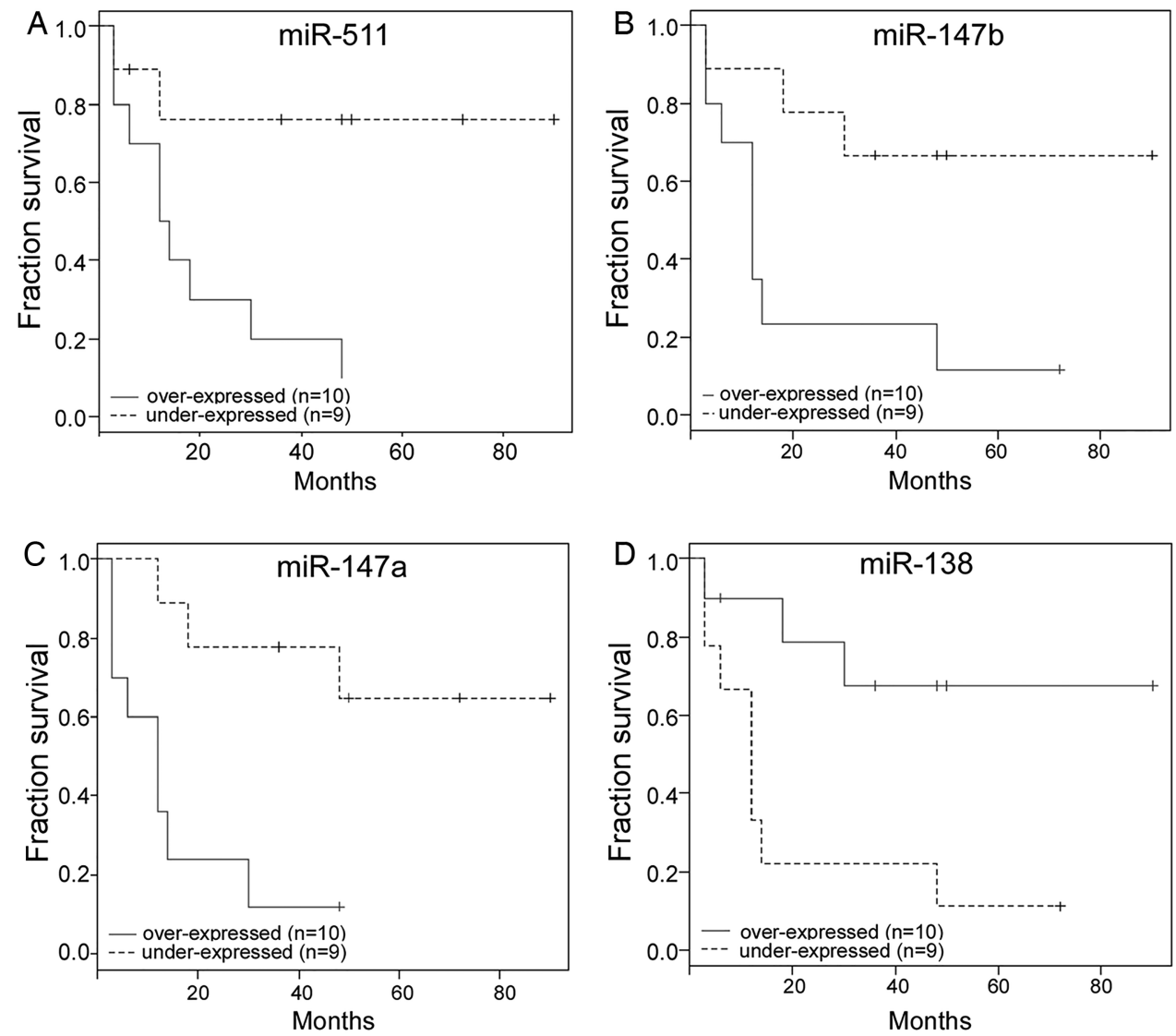

Figure 3 miRNAs are poor prognostic factors in hepatitis C virus (HCV)-associated diffuse large B-cell lymphoma (DLBCL). (A-D) HCV-associated DLBCL patients were categorised according to the miRNA level, and survival curves were plotted using the Kaplan-Meier method.

miR-25-5p levels were significantly correlated with low International Prognostic Index (IPI) (IPI 1 and 2). Finally, low miR-542-3p, miR-651-5p, miR-549a and high miR-369-5p levels were significantly associated with an advanced stage (stages III and IV). None of these correlations were found in non-infected DLBCL samples.

\section{Four miRNAs are correlated to the prognosis of HCV-associated DLBCL}

High levels of miR-138-5p were found to be correlated with significantly longer overall survival $(p=0.01, \quad H R=0.539)$, whereas, a shorter overall survival has been observed in patients with high expression of miR-511-5p $(p=0.012, H R=1.79)$, $\operatorname{miR}-147 \mathrm{a} \quad(\mathrm{p}=0.005, \quad \mathrm{HR}=1.46), \quad \operatorname{miR}-147 \mathrm{~b} \quad(\mathrm{p}=0.015$, $\mathrm{HR}=1.25$ ) (figure 3 ). None of these correlations were found in non-infected DLBCL samples.

\section{DISCUSSION}

This study analyses for the first time global miRNA expression profiling in HCV-positive DLBCLs. We found that HCV infection determines specific miRNA alterations, and that HCV-associated DLBCL have distinct molecular characteristics correlated with histopathological parameters and prognosis.

The only study which is known to have investigated the role of miRNA in HCV-associated lymphoma is the one of Peveling-Oberhag et al. They observed a reduced miR-26b expression in $\mathrm{HCV}$-positive patients with splenic marginal zone lymphoma (SMZL), a rare low-grade B-cell lymphoma. ${ }^{16}$

Although, it is difficult to attribute any discovered dysregulation of miRNA expression exclusively to $\mathrm{HCV}$ infection using indirect approach, our analysis identified 52 deregulated miRNAs that defines HCV-positive DLBCL. Different studies compared miRNA profiles of DLBCL with reactive lymph nodes, follicular lymphoma or Burkitt lymphoma revealing numerous and completely different altered miRNAs ${ }^{17}$; this discrepancy may be explained with the heterogeneity of DLBCL, different samples used to compare, or different technologies used, and HCV infection.

Among these 52 miRNA, miR-122 is under-expressed in $\mathrm{HCV}$-associated DLBCL compared to activated B cells in HCV patients. Such miR-122 levels are frequently reduced in HCC compared with normal liver. ${ }^{18}$ Moreover, many studies observed that miR-122 is highly expressed in liver and that it positively regulates HCV replication in liver cells. ${ }^{18}$ Interestingly miR-122 has been found to be over-expressed in germinal-centre B cells in patients with $\mathrm{HCV}$ infection with respect to patients without infection (data no shown).

miRNA Target prediction revealed interesting miRNA/gene interactions that could shed some light on HCV-associated lymphomagenesis. For example, miR-519b-3p and miR-96-5p, which are upregulated in our series of HCV-associated DLBCL, target CDKN1A, a gene involved in PI3K-Akt pathway, deregulated in many cancers. ${ }^{19}$ Moreover, we identified miR-20b-3p, miR-363-5p, miR-424-3p differentially expressed in HCV-associated DLBCL compared with DLBCL and $\mathrm{HCV}$-associated germinal centre B cells. It should be noted that all these three miRNAs, map to the chromosome Xq26 region. Although copy number changes targeting this chromosome are common in DLBCL, no candidate target genes have been consistently ascribed to these abnormalities. ${ }^{20}$ 
Finally, our study suggests that miR-138, miR-147a, miR-147b and miR-511 could be used to predict overall survival in HCV-associated DLBCL. miR-138 Might act as a tumour suppressor; in fact, reduced expression of miR-138 has been observed in different types of cancers ${ }^{21}$; ectopic expression of this miRNA reverse multidrug resistance, reduce tumour growth and invasion of cancer cells, ${ }^{22}$ probably through the regulation of focal adhesion kinase (FAK) expression. ${ }^{23}$

Interestingly, miR-511 and miR-147 are independently associated with toll-like receptor and inflammatory responses. miR-511 Is highly expressed in macrophages and dendritic cells with strong immunoregulatory function, because this miRNAs has a putative positive regulator of Toll-like Receptor $4 .^{24}$ In a mouse model, miR-147 is induced in macrophages after Toll-like receptor stimulation, and can negatively regulate activation of inflammatory pathways in myeloid cells. ${ }^{25}$

Moreover, the observed association does not allow the conclusion that the deregulated miRNAs might be driving factors in pathogenesis of HCV-DLBCL. Future analysis could explain the role of these miRNAs in $\mathrm{HCV}$-associated DLBCL tumorigenesis.

\section{Take home messages}

- Hepatitis C virus (HCV)-associated diffuse large B-cell lymphoma (DLBCL) have a specific miRNA signature.

- In HCV-associated DLBCL, distinct miRNA alterations are correlated with clinical and pathological parameters pathological parameters and prognosis.

- miRNA are potentially useful as prognostic or therapeutics biomarkers in HCV-associated DLBCL.

\begin{abstract}
Author affiliations
${ }^{1}$ Department of Pathophysiology and Transplantation, University of Milan, Milan, Italy

${ }^{2}$ Hematopathology Section, Division of Pathology, Department of Pathophysiology and Transplantation, University of Milan, IRCCS Ca' Granda-Maggiore Policlinico Hospital Foundation, Milan, Italy

${ }^{3}$ Division of Pathology, San Paolo Hospital, Milan, Italy

${ }^{4}$ Division of Pathology, A.Manzoni Hospital, Lecco, Italy

${ }^{5}$ Department of Laboratory Medicine, Niguarda Ca' Granda Hospital, Milan, Italy

${ }^{6}$ Division of Pathology, IRCCS Ca' Granda-Maggiore Policlinico Hospital Foundation, Milan, Italy

${ }^{7}$ Division of Hematology, Department of Clinical and Community Sciences, University of Milan, IRCCS Ca' Granda-Maggiore Policlinico Hospital Foundation, Milan, Italy ${ }^{8}$ Division of Pathology, Department of Pathophysiology and Transplantation, University of Milan, IRCCS Ca' Granda-Maggiore Policlinico Hospital Foundation, Milan, Italy
\end{abstract}

Contributors Case selection and collecting data: $A M, E B, M G, L B$ and $S B$; data/ results analysis: CA, VV and FS; manuscript: CA, UG and FS.

Competing interests None.

Patient consent Obtained.

Ethics approval This study was approved by Institutional Review Board of IRCCS Ca' Granda - Maggiore Policlinico Hospital Foundation.

Provenance and peer review Not commissioned; externally peer reviewed.

\section{REFERENCES}

1 Peveling-Oberhag J, Arcaini L, Hansmann ML, et al. Hepatitis C-associated B-cell non-Hodgkin lymphomas. Epidemiology, molecular signature and clinical management. J Hepatol 2013;59:169-77.

2 De Re V, Caggiari L, Garziera M, et al. Molecular signature in HCV-positive lymphomas. Clin Dev Immunol 2012;2012:623465. http://dx.doi.org/10.1155/2012/ 623465

3 Marcucci F, Mele A. Hepatitis viruses and non-Hodgkin lymphoma: epidemiology, mechanisms of tumorigenesis, and therapeutic opportunities. Blood 2011;117:1792-8.

4 Alizadeh AA, Eisen MB, Davis RE, et al. Distinct types of diffuse large B-cell lymphoma identified by gene expression profiling. Nature 2000; 403:503-11.

5 Bea S, Zettl A, Wright G, et al. Lymphoma/Leukemia Molecular Profiling Project. Diffuse large B-cell lymphoma subgroups have distinct genetic profiles that influence tumor biology and improve gene-expression-based survival prediction. Blood 2005; 106:3183-90.

6 Viswanatha DS, Dogan A. Hepatitis C virus and lymphoma. J Clin Pathol 2007;60:1378-83.

7 Besson C, Canioni D, Lepage $E$, et al. Characteristics and outcome of diffuse large B-cell lymphoma in hepatitis C virus- positive patients in LNH93 and LNH98 groupe d' etude des lymphomes de l'adulte programs. J Clin Oncol 2006;24:953-60

8 Augello C, Vaira V, Caruso L, et al. MicroRNA profiling of hepatocarcinogenesis identifies C19MC cluster as a novel prognostic biomarker in hepatocellular carcinoma. Liver Int 2012;32:772-82.

9 Skalsky RL, Cullen BR. Viruses, microRNAs, and host interactions. Annu Rev Microbiol 2010;64:123-41.

10 Lung RW, Tong JH, To KF. Emerging roles of small Epstein-Barr virus derived non-coding RNAs in epithelial malignancy. Int J Mol Sci 2013; 14:17378-409.

11 Jardin F, Figeac M. MicroRNAs in lymphoma, from diagnosis to targeted therapy. Curr Opin Oncol 2013;25:480-6.

12 Mazan-Mamczarz K, Gartenhaus RB. Role of microRNA deregulation in the pathogenesis of diffuse large B-cell lymphoma (DLBCL). Leuk Res 2013; $37: 1420-8$

13 Swerdlow SH, Campo E, Harris NL, et al. WHO classification of tumours of haematopoietic and lymphoid tissues. Lyon, France: IARC Press, 2008.

14 Vaira V, Faversani A, Dohi T, et al. miR-296 regulation of a cell polarity-cell plasticity module controls tumor progression. Oncogene 2012;31:27-38.

15 Vergoulis T, Vlachos IS, Alexiou P, et al. Tarbase 6.0: capturing the exponential growth of miRNA targets with experimental support. Nucl Acids Res 2012;40: D222-9.

16 Peveling-Oberhag J, Crisman G, Schmidt A, et al. Dysregulation of global microRNA expression in splenic marginal zone lymphoma and influence of chronic hepatitis $C$ virus infection. Leukemia 2012;26:1654-62.

17 Mazan-Mamczarz K, Gartenhaus RB. Role of microRNA deregulation in the pathogenesis of diffuse large B-cell lymphoma (DLBCL). Leuk Res 2013;37:1420-8.

18 Jopling C. Liver-specific microR-122: Biogenesis and function. RNA Biol 2012;9:137-42.

19 Kawauchi K, Ogasawara T, Yasuyama M, et al. The PI3K/Akt pathway as a target in the treatment of hematologic malignancies. Anticancer Agents Med Chem 2009:9:550-9.

20 Beà S, Colomo L, López-Guillermo A, et al. Clinicopathologic significance and prognostic value of chromosomal imbalances in diffuse large B-cell lymphomas. J Clin Oncol 2004;22:3498-506.

21 Wang W, Zhao LJ, Tan YX, et al. MiR-138 induces cell cycle arrest by targeting cyclin D3 in hepatocellular carcinoma. Carcinogenesis 2012; 33:1113-20.

22 Zhao $\mathrm{X}$, Yang L, Hu J, et al. miR-138 might reverse multidrug resistance of leukemia cells. Leuk Res 2010;34:1078-82.

23 Golubovskaya VM, Sumbler B, Ho B, et al. MiR-138 and MiR-135 directly target focal adhesion kinase, inhibit cell invasion, and increase sensitivity to chemotherapy in cancer cells. Anticancer Agents Med Chem 2014;14:18-28.

24 Tserel L, Runnel T, Kisand K, et al. MicroRNA expression profiles of human blood monocyte-derived dendritic cells and macrophages reveal miR-511 as putative positive regulator of Toll-like receptor 4. J Biol Chem 2011; 286:26487-95.

25 Liu G, Friggeri A, Yang Y, et al. miR-147, a microRNA that is induced upon Toll-like receptor stimulation, regulates murine macrophage inflammatory responses. Proc Natl Acad Sci U S A 2009;106:15819-24. 